ARTICLE

https://doi.org/10.1038/s41467-019-13062-z

\title{
A fln-2 mutation affects lethal pathology and lifespan in C. elegans
}

Yuan Zhao (1) ${ }^{1}$, Hongyuan Wang (10 ${ }^{1,2}$, Richard J. Poole $\mathbb{1}^{3}{ }^{3} \&$ David Gems ${ }^{1 *}$

Differences in genetic background in model organisms can have complex effects on phenotypes of interest. We previously reported a difference in hermaphrodite lifespan between two wild-type lines widely used by $C$. elegans researchers (N2 hermaphrodite and male stocks). Here, using pathology-based approaches and genome sequencing, we identify the cause of this difference as a nonsense mutation in the filamin gene $f(n-2$ in the male stock, which reduces early mortality caused by pharyngeal infection. We show how fln-2 variation explains previous discrepancies involving effects of sir-2.1 (sirtuin deacetylase) on ageing, and show that in a fln-2(+) background, sir-2.1 over-expression causes an FUDR (DNA synthesis inhibitor)-dependent reduction in pharyngeal infection and increase in lifespan. In addition we show how fIn-2 variation confounds effects on lifespan of daf-2 (insulin/IGF-1 signalling), daf-12 (steroid hormone signalling), and eat-2 (putative dietary restriction). These findings underscore the importance of identifying and controlling genetic background variation.

\footnotetext{
${ }^{1}$ Institute of Healthy Ageing, Department of Genetics, Evolution and Environment, University College London, London WC1E 6BT, UK. ${ }^{2}$ School of Chemistry and Chemical Engineering, Harbin Institute of Technology, Harbin, China. ${ }^{3}$ Department of Cell and Developmental Biology, University College London, London WC1E 6BT, UK. *email: david.gems@ucl.ac.uk
} 
$\mathrm{R}$ esearch using invertebrate model organisms has revealed much about how genotype specifies many aspects of phenotype, including ageing. Studies on the short-lived nematode Caenorhabditis elegans, in particular, have led to the discovery of signalling pathways that affect lifespan, not only in nematodes but also in higher organisms, including humans ${ }^{1,2}$. These include the insulin/IGF-like signalling (IIS) and the target of rapamycin (TOR) pathways ${ }^{3,4}$. In recent years, the importance of genetic variation in C. elegans strain backgrounds has become increasingly clear, partly arising from inadvertent selection acting on the wild type under laboratory conditions and/or random mutation accumulation ${ }^{5-7}$. Such variation may confound the effects of genes of interest.

First isolated more than 60 years ago, the laboratory strain N2 (Bristol) is the standard reference strain for C. elegans research ${ }^{6}$. $\mathrm{N} 2$ is distributed by the Caenorhabditis Genetics Center (CGC) either as a hermaphrodite only stock (which we will refer to here as $\mathrm{N} 2 \mathrm{H}$ ) or as a male stock (referred to at the CGC as N2 male, which we will refer to as N2M). We previously noted that hermaphrodite lifespan is slightly but consistently longer in N2M than $\mathrm{N} 2 \mathrm{H}(+11 \% \text { median lifespan })^{8}$. As both stocks have been used as wild type, it is possible that the stock difference may have confounded prior studies of gene effects on lifespan.

Ageing limits animal lifespan by promoting multiple pathologies that increase mortality rate. Using necropsy analysis, we recently identified death with a swollen, infected pharynx ( $\mathrm{P}$ [big $\mathrm{P}$ ] death) as one form of mortality in C. elegans ${ }^{9}$. Under standard laboratory culture conditions ${ }^{10}$, initial intra-pharyngeal infection occurs in early adulthood during a period of fast pumping and mastication of the bacterial food source, and later accounts for $\sim 40 \%$ of $\mathrm{N} 2 \mathrm{H}$ deaths, largely those occurring prior to mean lifespan. The remainder of the population die with an uninfected but often atrophied pharynx ( $\mathrm{p}$ [small p] death). Changes in the shape of survival curves of different $C$. elegans strains can be understood in terms of altered frequency and/or timing of $\mathrm{P}$ and $\mathrm{p}$ death (mortality deconvolution) ${ }^{9}$.

In this study, we use mortality deconvolution to investigate the cause of the difference in lifespan between $\mathrm{N} 2 \mathrm{H}$ and $\mathrm{N} 2 \mathrm{M}$, and the question of which of these $\mathrm{N} 2$ lines is wild type. We report that the longer lifespan of $\mathrm{N} 2 \mathrm{M}$ is attributable to reduced $\mathrm{P}$ frequency that results from a single nonsense mutation in the $\mathrm{X}$-linked gene $f l n-2$, indicating that $\mathrm{N} 2 \mathrm{H}$ is wild type and $\mathrm{N} 2 \mathrm{M}$ mutant. We demonstrate the importance of controlling for $f l n-2$ genotype by illustrating how it can confound investigations, using several examples of genes that have been the focus of many studies of ageing in C. elegans: sir-2.1, daf-2, daf-12 and eat-2.

\section{Results}

N2M longevity is due to reduced $P$ death frequency. To investigate the causes of the lifespan difference between $\mathrm{N} 2 \mathrm{H}$ and N2M, we performed mortality deconvolution on the two lines, comparing timing and frequency of $\mathrm{P}$ and $\mathrm{p}$ death, using combined mortality and necropsy analysis ${ }^{9}$. Overall mean lifespan of N2M hermaphrodites was $16.6 \%$ longer than $\mathrm{N} 2 \mathrm{H}$ (22.1 days vs. 18.7 days, $p<0.0001$, log rank test)(Fig. 1a; Supplementary Table 1), consistent with previous findings ${ }^{8}$. Necropsy analysis revealed that $37 \%$ of $\mathrm{N} 2 \mathrm{H}$ worms died with $\mathrm{P}$, consistent with previous findings ${ }^{9}$, but this was the case in only $13 \%$ of $\mathrm{N} 2 \mathrm{M}$ worms $(p=0.0031$, Student's $t$ test)(Fig. 1b). By contrast, the lifespans of both the $\mathrm{P}$ and $\mathrm{p}$ subpopulations were similar in the two lines (Fig. 1a; Supplementary Table 1). Thus, the lifespan difference between $\mathrm{N} 2 \mathrm{H}$ and $\mathrm{N} 2 \mathrm{M}$ is attributable to reduced $\mathrm{P}$ death frequency in the latter. This predicts that blocking $E$. coli proliferation with antibiotics or UV radiation, which completely prevents $\mathrm{P}$ death ${ }^{9}$, should abrogate the difference in lifespan between $\mathrm{N} 2 \mathrm{H}$ and N2M, and this proved to be the case (Fig. 1a; Supplementary Fig. 1a; Supplementary Table 1).

To further investigate the difference between $\mathrm{N} 2 \mathrm{H}$ and $\mathrm{N} 2 \mathrm{M}$ we compared other characteristics of the two lines. No difference in self-fertilised brood size was detected, consistent with previous findings ${ }^{8}$ (Supplementary Fig. 1b). Mutants with reduced pumping rate often show reduced $\mathrm{P}$ frequency ${ }^{9}$, raising the possibility that pumping rate might be reduced in $\mathrm{N} 2 \mathrm{M}$, but it was not (Supplementary Fig. 1c). Moreover, the difference between $\mathrm{N} 2 \mathrm{H}$ and $\mathrm{N} 2 \mathrm{M}$ is not attributable to effects of parental mating in the latter: hermaphrodite progeny of mated $\mathrm{N} 2 \mathrm{H}$ still show higher frequency of P death than N2M (Supplementary Fig. 1d). Thus, N2M's intrinsic resistance to pharyngeal E. coli infection accounts for its longer lifespan.

We previously reported evidence that pharyngeal infection occurs in two stages: initial bacterial invasion occurs during early adulthood, apparently due to activity-dependent damage to the pharyngeal cuticle, while major infection occurs later in some animals when bacteria escape cyst-like intracellular structures and proliferate $^{9}$ (for examples of different stages of infection, see Supplementary Fig. 2; Supplementary Movie 1). To test whether each stage can be selectively suppressed, worms were fed with $E$. coli OP50 expressing a red fluorescent protein (OP50-RFP), which renders initial bacterial invasion visible as red fluorescent puncta within pharyngeal tissue ${ }^{9}$ (Fig. 1c). Culture on antibiotic-treated $E$. coli did not suppress initial bacterial invasion of the pharynx (presumably because antibiotics do not suppress grindergenerated mechanical damage and perforation of the pharyngeal cuticle $^{9}$ ) but did prevent later bacterial proliferation, while in pumping-defective phm-2 mutants initial invasion was fully suppressed (Fig. 1c), perhaps due to a combination of reduced mechanical damage and bacterial lawn avoidance ${ }^{11}$. Thus, it is possible to selectively suppress the invasion and proliferation stages.

We then asked: which stage is suppressed in N2M? Initial bacterial invasion was observed in $40 \%$ of the population in $\mathrm{N} 2 \mathrm{M}$, compared to $75 \%$ in $\mathrm{N} 2 \mathrm{H}$ as early as day 4 of adulthood; yet in both strains about half of those showing early signs of invasion die with a swollen, infected pharynx (Fig. 1d). These results suggest that $\mathrm{N} 2 \mathrm{M}$ is resistant to pharyngeal cuticle perforation and/or initial bacterial invasion rather than to later bacterial proliferation.

N2M longevity is caused by $\boldsymbol{f l n}$-2(ot611). Reduced $\mathrm{P}$ frequency in N2M likely reflects a different genotype. Analysis of F1 hybrids from a N2H/N2M cross showed that the N2M infrequent P trait is recessive (Fig. 1e). We also noted, during backcrossing with $\mathrm{N} 2 \mathrm{M}$ of a strain with high frequency of $\mathrm{P}$, that $\mathrm{P}$ frequency dropped in a single round of outcrossing from high to low $P$ frequency (Fig. 1f), suggesting effects of a single gene.

To identify the causative variant, whole genome sequencing was used. An initial comparison between genomic sequences of the two N2 lines revealed 4259 single nucleotide polymorphisms (SNPs) and small insertions and deletions (INDELs). Most of these mutations likely accumulated during separate propagation of $\mathrm{N} 2 \mathrm{H}$ and N2M before they were both frozen in $1975^{8}$. In order to map the mutation affecting $\mathrm{P}$ frequency, we performed Variant Discovery Mapping ${ }^{12}$. For this, N2H and N2M were crossed and the progeny of individual F2 recombinant lines with the N2M phenotype pooled and subjected to genome sequencing (Fig. 2a). Parental allele enrichment peaked near the $10 \mathrm{Mb}$ position on LGX implying the presence of the causal variant in this vicinity (Fig. 2b, c; Supplementary Figs. 3 and 4a). Variants within this region co-segregated with low $\mathrm{P}$ frequency in the aforementioned 
a

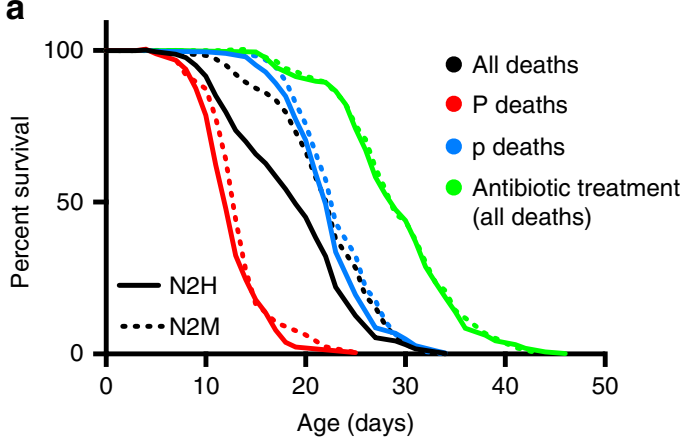

C

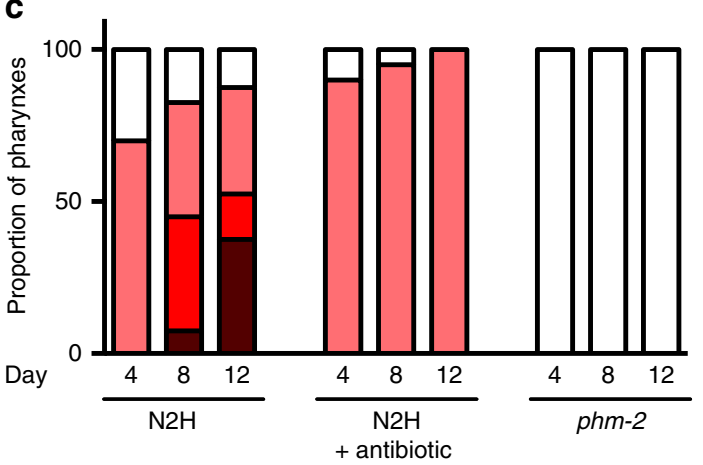

b

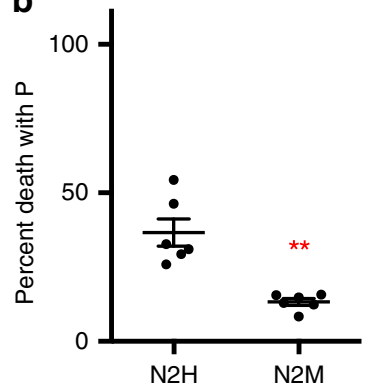

d

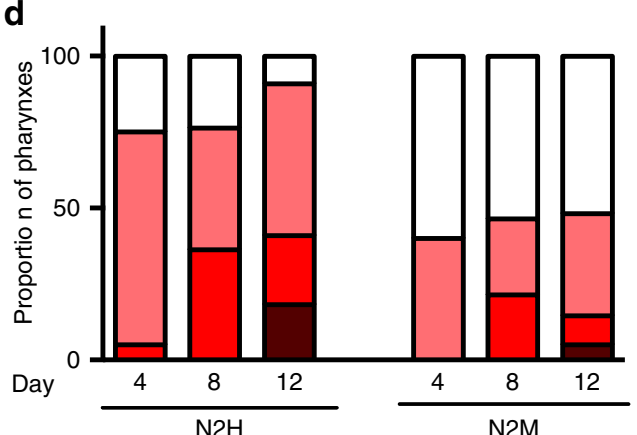

f

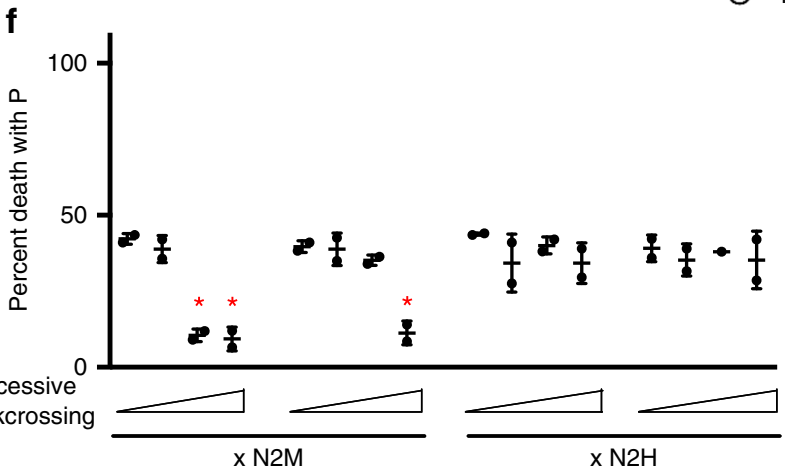

e

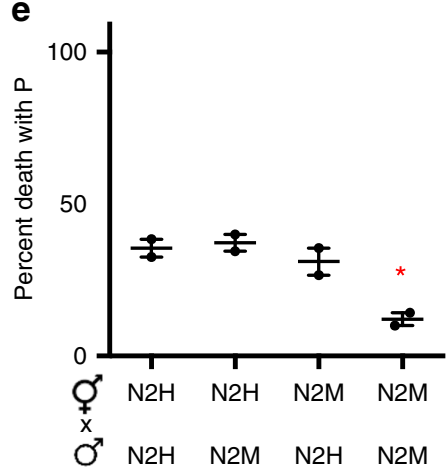

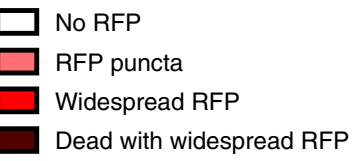

Dead with widespread RFP 


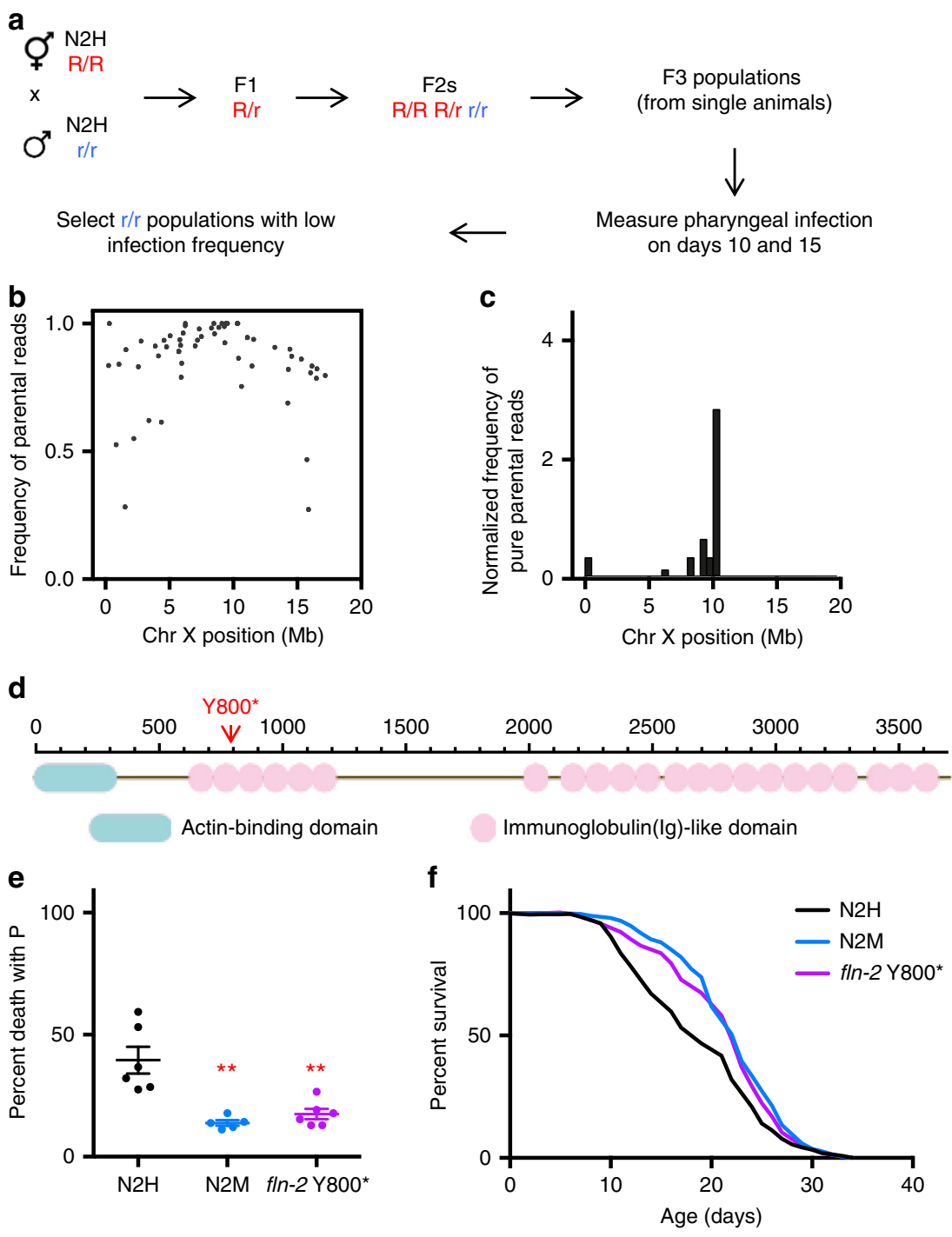

Fig. 2 Resistance to $P$ death is caused by a recessive mutation in fln-2. a Design of Variant Discovery Mapping strategy to select the recessive low $P$ phenotype of N2M in F3 progeny. $\mathbf{b}$ Frequency of parental (N2M genotype) reads on chromosome $X$. For results for all chromosomes see Supplementary Fig. 3. c Normalised frequency of pure parental (N2M genotype) reads on chromosome X. $\mathbf{d}$ Illustration of the fln-2(ot611) mutation on FLN-2A, the longest isoform of fln-2. e Necropsy analysis and $\mathbf{f}$ lifespan of $\mathrm{N} 2 \mathrm{H}$-derived fln-2(syb202) Y800* strain. N2H survival curve in black, N2M in blue, and fln-2 Y800* in purple. Asterisks indicate $p<0.01$, Student's $t$ test. Error bars represent s.e.m. of three trials. See Supplementary Table 2 for full statistics. See Supplementary data 1 for raw mortality data. Source data are provided as a Source Data file

backcrossed strains (Fig. 1f), as established by sequencing (16 strains sequenced). The $3 \mathrm{Mb}$ region near the parental allele enrichment peak contained four genetic differences predicted to alter protein sequence, including three missense and one nonsense mutation (Supplementary Fig. 4b). The nonsense mutation was in $f l n-2$, which encodes a filamin that is localised, among other places, to the pharyngeal cuticle ${ }^{13}$. We therefore focused on $\mathrm{fln}-2$.

FLN-2 is a distant ortholog of filamin-A (FLNA) in humans, which links actin filaments to membranes and is involved in cytoskeletal remodelling and mechanical force sensing. The variant in $f l n-2$ results in a stop codon at Y800 in N2M, downstream of the $\mathrm{N}$-terminal actin-binding domain in the second immunoglobulin(Ig)-like domain ${ }^{13}$ (Fig. 2d). Of the 16 isoforms that $f l n-2$ encodes, the premature stop codon affects six, all of which contain the actin-binding domain (Supplementary Fig. 4c). We checked the $f l n-2$ allele in other C. elegans wild isolates available at the Caenorhabditis elegans Natural Diversity Resource $(\mathrm{CeNDR})^{14}$, and found that none of the wild strains contain the Y800* allele. $f l n-2$ orthologs in a selection of other nematode species also encode the full-length $f \ln -2(+)$ allele and not the Y800* equivalent (Supplementary Fig. 4d). We therefore conclude that the $f l n-2$ variant in $\mathrm{N} 2 \mathrm{M}$ is a nonsense mutation. This mutation was previously observed 6 out of 17 sequenced EMS-mutagenized genomes ${ }^{15}$ and designated $f l n-2$ (ot611) (formerly $f \ln b-1)$; on the assumption that strains containing the mutant allele were derived from $\mathrm{N} 2 \mathrm{M}$, we will refer to the variant identified here as $f \ln -2$ (ot611).

To test whether $f l n-2$ (ot611) causes the N2M infrequent P trait, we first tested effects of the mutation $f l n-2$ (ok1305), which contains a deletion in the $\mathrm{N}$-terminal region. Although it showed infrequent P death (Supplementary Fig. 5a), the strain RB1240 also contained ot611, making this result difficult to interpret. Another $f l n-2$ mutant allele, $f l n-2(t m 4687)$, which does not contain ot611, has a deletion in the C-terminal region affecting all $f l n-2$ isoforms. This also caused low P frequency (Supplementary Fig. 5b), demonstrating that variation at $f l n-2$ can affect $P$ incidence. Additionally, we introduced the $\mathrm{Y}^{8} 80^{*}$ mutation into 
$\mathrm{N} 2 \mathrm{H}$ using CRISPR/Cas9. The resulting $f \ln -2(s y b 202) \mathrm{Y} 800^{*}$ allele led to recapitulation of the N2M phenotype, reducing P frequency from 45 to $19 \%$, with little effect on p lifespan (Fig. 2e, f; Supplementary Table 2). Taken together, these results indicate that $f \ln -2$ (ot611) is the cause of the reduced P frequency of N2M. Tests on four C. elegans wild isolates showed a P death incidence similar to that of $\mathrm{N} 2 \mathrm{H}$ (Supplementary Fig. 5c), suggesting that this relatively high $\mathrm{P}$ frequency is a species-typical (i.e. wild-type) characteristic of C. elegans.

Although $\mathrm{N} 2 \mathrm{H}$ is predominantly used as the wild-type strain in C. elegans research (requested from the CGC 3,633 times compared to 173 times for N2M [2006-2016]), N2M is sometimes used for strain construction or backcrossing. As a limited test of the presence of $f \ln -2$ (ot611) among C. elegans strains, we genotyped the $f l n-2$ allele in a selection of strains present in our strain collection. This revealed the presence of $f l n-2$ (ot611) in 23/ 50 of cases, including some strains generated by the C. elegans Gene Knockout Project ( $g k$ and $o k$ alleles)(ot611 present: VC prefix strains, 6/10; RB prefix strains, 6/6) and the $C$. elegans Expression Project ( $s$ alleles)(ot611 present: 9/9) (Supplementary Table 3).

Discrepant effect of $s i r-2.1$ attributable to $\boldsymbol{f l n}$-2 variation. Given the effect of the $f l n-2$ (ot611) allele on pharyngeal pathology and lifespan, and the fact that the Gems lab routinely used N2M as the wild-type for backcrossing ${ }^{8}$, we set out to revisit some earlier, puzzling results. In budding yeast (Saccharomyces cerevisiae) over-expression of the NAD-dependent histone deacetylase SIR2 (sirtuin) increases replicative lifespan ${ }^{18}$. There have been conflicting reports about whether or not increasing sirtuin levels in $C$. elegans increases lifespan. In C. elegans, independent transgenic lines with sir-2.1 over-expression (oe) generated in two studies were long lived ${ }^{19,20}$. In the case of one high copy number sir-2.1 over-expresser (transgene array geIs3, hereafter referred to as sir$2.1(o e))^{19}$ it was subsequently reported that longevity of the original strain (LG100) was attributable to a second site $d y f$ gene mutation, and that sir-2.1(oe) no longer increased lifespan after backcrossing with $\mathrm{N}_{2} \mathrm{M}^{21}$. However, in another study that confirmed the presence and action of the second site mutation, a residual, smaller lifespan-increasing effect of sir-2.1(oe) was seen after outcrossing with N2 (line not specified) ${ }^{22}$.

We asked: could this discrepancy be attributable to effects of $\mathrm{N} 2 \mathrm{H}$ vs. N2M background differences? Genotyping of the strains used by Viswanathan and Guarente (2011) ${ }^{22}$ and Burnett et al. ${ }^{21}$ confirmed that the former were $f \ln -2(+)$, and the latter $f \ln -2($ ot611), presumably reflecting the different $\mathrm{N} 2$ lines used in backcrossing of the original sir-2.1(oe) line. Consistent with both previous reports, sir-2.1(oe) resulted in increased lifespan in a $f l n-2(+)$ background $(+27 \%$ mean lifespan, $p<0.0001$, log rank test), but not a $f h-2$ (ot611) background ( $p=0.96, \log$ rank test) (Fig. 3a; Supplementary Fig. 6a; Supplementary Table 4) under culture conditions as previously described, in which the thymidylate synthase inhibitor 5 -fluorodeoxyuridine (FUDR) is added to block progeny development (see Methods and below).

Mortality deconvolution revealed that there was no significant difference in the frequency of $\mathrm{P}$ death in a fln-2(ot611) background (GA strains: GA468 geIs3 [sir-2.1(+) rol-6]; $f l n-2$ (ot611) compared to GA707 wuEx166 [rol-6]; fln-2(ot611)), but that sir-2.1(oe) significantly reduced P death frequency in a $f l n-2$ (+) background (LG strains: LG394 geIs3 [sir-2.1(+) rol-6] compared to LG398 geIs101 [rol-6]) (Fig. 3b; Supplementary Fig. 6b). All four strains were then backcrossed with N2H, which for the GA strains replaced $f l n-2(o t 611)$ with $f l n-2(+)$, after which sir-2.1(oe) reduced P death frequency and increased lifespan in both sets of strains $(+19 \%$ and $+32 \%$ mean lifespan, respectively)
(Fig. 3c, d; Supplementary Table 5). In a $f l n-2(+)$ background, the increase in lifespan resulting from sir-2.1(oe) was the result not only of reduced $\mathrm{P}$ death frequency, but also of a small but statistically significant increase in $p$ lifespan $(+14 \%,+13 \%$ mean lifespan in GA strains and LG strains, $p=0.0016$ and 0.0002 , respectively, log rank test) (Supplementary Fig. 6c; Supplementary Table 5).

In the previous studies, FUDR was used to block progeny development: $1.6 \mathrm{mM}$ in the 2001 study $^{19}$, and 10 to $50 \mu \mathrm{M}^{21}$ or an unspecified concentration ${ }^{22}$ in the 2011 studies. Therefore in the trails described above FUDR was included $(10 \mu \mathrm{M}$ in Fig. 3a-d, and $50 \mu \mathrm{M}$ in Supplementary Fig. 6a, b). Since impacts of many interventions on lifespan are affected by the presence of FUDR $^{23-26}$, and since fluoropyrimidines have complex effects both on E. coli and C. elegans ${ }^{27}$, we also tested effects of sir-2.1(oe) on $\mathrm{P}$ frequency and lifespan (overall and $\mathrm{P}$ or $\mathrm{p}$ alone) in the absence of FUDR. Here no effect was detected in either $f l n-2$ background, in either the original strains or the outcrossed strains (2 trials each) (Fig. 3e, f; Supplementary Fig. 6d, e; Supplementary Tables 6 and 7).

We further tested for interaction between FUDR and sir-2.1 over-expression using a previously described low copy number transgene array pkIs $1642^{20}$. It was previously reported that longevity resulting from pkIs1642 sir-2.1(oe) was abrogated by outcrossing ${ }^{21}$. Here both original and outcrossed strains were $f l n-2(+)$. Nonetheless, we observed that the longevity of the nonoutcrossed pkIs1642 strain was, again, FUDR dependent (Supplementary Fig. 7; Supplementary Table 8). However, here longevity resulted from increased $\mathrm{p}$ lifespan rather than reduced P frequency. For more details, see Supplementary Fig. 6.

We also asked whether loss of sir-2.1 increases P frequency, using the sir-2.1(ok434) null allele that harbours a deletion that disrupts the sirtuin catalytic domain ${ }^{28,29}$. After backcrossing into a $f \ln -2(+)$ background, sir-2.1(ok434) reduced overall lifespan independently of FUDR $(-8 \%$ and $-9 \%$ mean lifespan, $p=$ 0.0043 and 0.0425 , with and without FUDR respectively, log rank test), consistent with previous reports ${ }^{20,28}$. However, the manner in which sir-2.1(ok434) shortened lifespan was FUDR dependent: with FUDR it increased $P$ death frequency, but without FUDR it reduced $p$ lifespan (Supplementary Fig. 8a, b; Supplementary Table 9). Thus, again, the impact of sir-2.1 on worm ageing is FUDR dependent.

The FUDR dependence of the reduced P death caused by sir-2.1 (oe) suggests that SIR-2.1 and FUDR act synergistically to protect against bacterial infection of the pharynx. To explore the mechanism of this protection we compared the progression of pharyngeal infection in OP50-RFP-fed worms with either sir-2.1 (oe), FUDR, or both. Without FUDR, as expected, sir-2.1(oe) had little effect on either the initiation or subsequent spread of $E$. coli infection; with FUDR, the appearance of intracellular RFP puncta was unaffected by sir-2.1(oe), but the progression of infections in older animals was suppressed, with fewer animals showing widespread RFP (Fig. 3g). Notably, FUDR alone delayed the appearance of RFP puncta, though it had little effect on the frequency of P (Fig. 3g). Thus, FUDR and sir-2.1(oe) affect pharyngeal infection by different mechanisms: FUDR delays initial invasion, while SIR-2.1 suppresses later spread of infection. This suggests that FUDR-induced resistence to bacterial pathogenicity enables SIR-2.1 to reduce later E. coli proliferation, leading to synergy between the two interventions. Consistent with this, pretreatment of $C$. elegans with FUDR increases resistance to the Gram-negative bacterial pathogen Pseudomonas aeruginosa ${ }^{30}$.

$f l n-2$ variation confounds effects of $d a f-12$ on lifespan. The daf12 gene encodes a steroid hormone receptor, and has been shown 

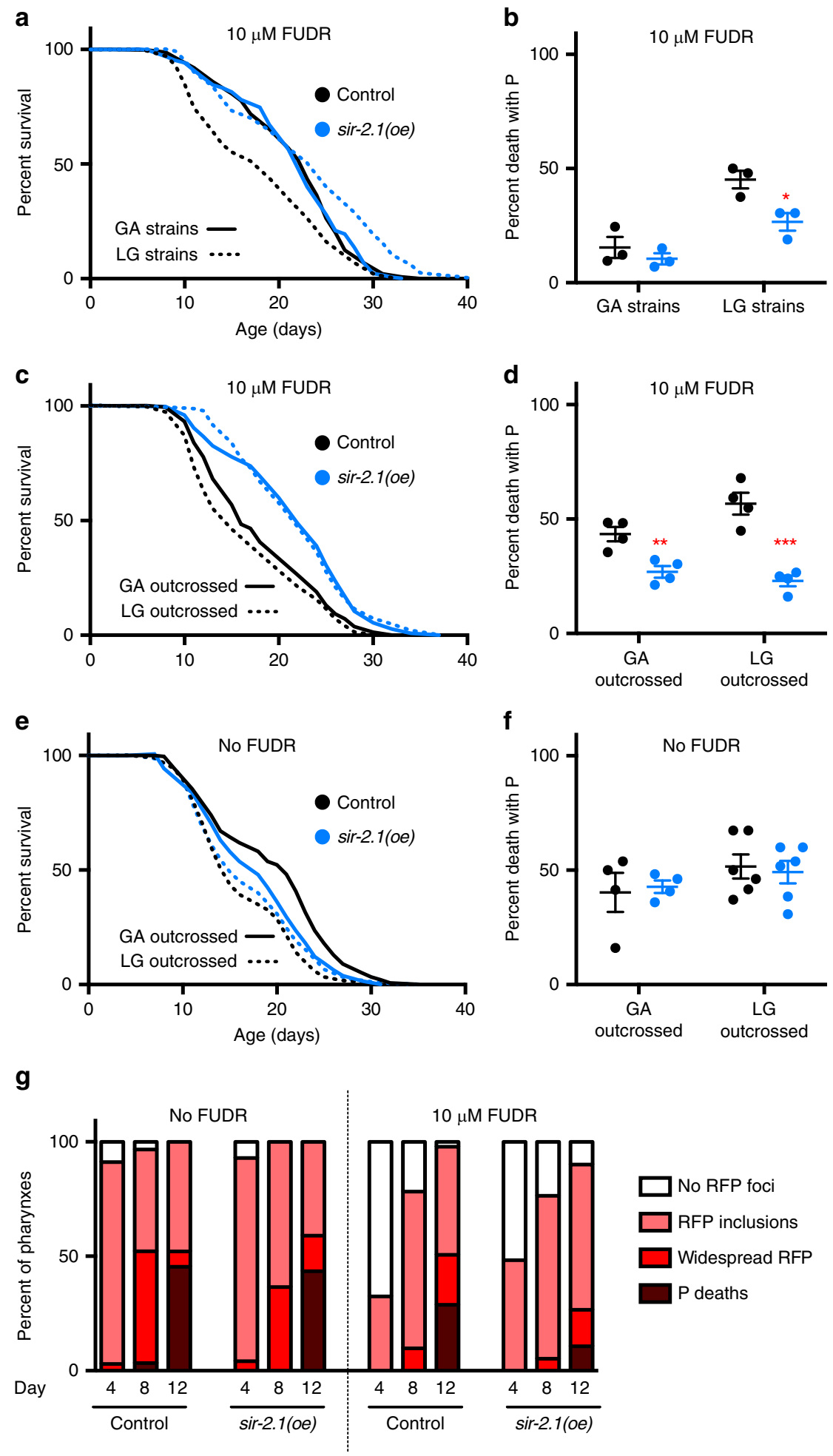

to have complex effects on different $d a f-2$ insulin/IGF-1 receptor mutants $^{31-33}$. For example, daf-2(m41) longevity (the Age phenotype) is partially suppressed by daf-12(m20) under certain conditions $^{31}$. We noted that daf-12(m20) shortens daf-2(m41) lifespan by increasing early mortality ${ }^{31}$, and that the daf- 12 and $f l n-2$ loci are closely linked on LGX (at X:2.36 $\pm 0.007 \mathrm{cM}$ and $\mathrm{X}: 1.18 \pm 0.015 \mathrm{cM}$, respectively). This raised the possibility that some observed effects of daf-12 on daf-2 Age might be attributable to the differences in $\mathrm{P}$ frequency due to variation at the $f l n-2$ locus.
We genotyped daf-2(m41) and daf-2(m41); daf-12(m20) double mutant strains used in earlier studies ${ }^{31,32}$, and found that the daf$2(m 41)$ strain, but not the daf-2; daf-12 strain, contained $f l n-2$ (ot611). Consistent with previous findings ${ }^{31}$, daf-2; daf-12 lifespan was shorter than daf-2; $f l n-2$; this was due mainly to a 9-fold higher $\mathrm{P}$ frequency in daf-2; daf-12 (Fig. 4a, b). Comparison of daf-2; daf-12 and daf-2 after removal of $f l n-2$ (ot611) by outcrossing showed a much smaller but statistically significant $60 \%$ increase in P frequency (Fig. 4b). Notably, daf-12 had little effect on daf-2(m41) p lifespan (Fig. 4c); moreover, fln-2 
Fig. 3 Reduction of $\mathrm{P}$ death by fln-2(ot611) masks effects of high copy number sir-2.1 over-expression (oe) on lifespan. a Lifespan and $\mathbf{b}$ necropsy analysis of sir-2.1(oe) and control strains used in previous studies. GA strains are indicated by solid lines: GA468 gels3 sir-2.1(oe); fln-2(ot611) (blue) and GA707 wuEx166; fln-2(ot611) control (black). LG strains are indicated by dashed lines: LG394 gels3 sir-2.1(oe) (blue) and LG398 gels101 control (black). sir-2.1(oe) reduced $P$ death and extended lifespan in LG strains but not GA strains, consistent with both previous, conflicting studies 21,22 . Error bar represents s.e.m. of three trials. See Supplementary Table 4 for full statistics. See Supplementary data 1 for raw mortality data. Source data are provided as a Source Data file. c Lifespan and $\mathbf{d}$ necropsy analysis of sir-2.1(oe) and control strains after backcrossing four times with N2H. GA outcrossed in solid lines: GA1909 gels3 sir2.1(oe) (blue) and GA1907 wuEx166 control (black); LG outcrossed in dashed lines: GA1913 gels3 sir-2.1(oe) (blue) and GA1915 gels101 control (black). sir-2.1 (oe) strains (blue) from both labs showed a reduced frequency of $\mathrm{P}$ death compared to control strains (black) in fln-2(+) background. See Supplementary Table 5 for full statistics. a-d Trials conducted in the presence of $10 \mu$ M FUDR. See Supplementary data 1 for raw mortality data. e Lifespan and $\mathbf{f}$ necropsy analysis of backcrossed sir-2.1(oe) and control strains, with FUDR excluded. Colour coding as in (c, d). See Supplementary Table 7 for full statistics. See Supplementary data 1 for raw mortality data. $\mathbf{g}$ sir-2.1(oe) and $10 \mu \mathrm{M}$ FUDR interact to protect against progression of bacteria infection. Pharyngeal invasion and widespread infection were scored in worms fed RFP-expressing bacteria. Colour coding as in Fig. 1c. Mean of two trials, $n=16-20$ per time point in each trial
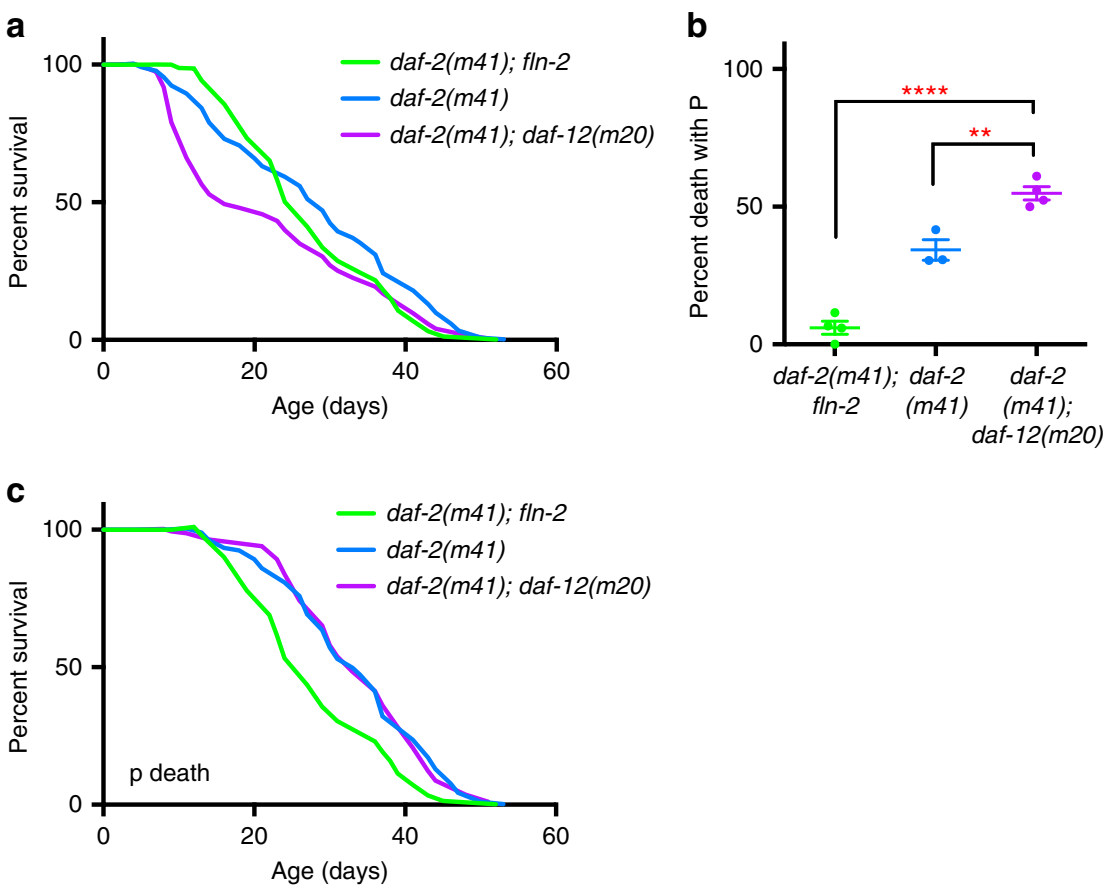

Fig. 4 Effect of daf- 12 on daf- 2 at $25^{\circ} \mathrm{C}$ is partially affected by fln-2(ot611) variation. a Lifespan and $\mathbf{b}$ necropsy analysis of daf-2(m41); daf-12(m20) and daf-2 (m41) mutants in fln-2(+) or fln-2(ot611) backgrounds. daf-2 fln-2 in green, daf-2 in blue, and daf-2 daf-12 in purple. Asterisks indicate significant differences, $p<0.0001$ and 0.01, respectively. Error bars represent s.d. of two trials. See Supplementary Table 10 for full statistics, and Supplementary data 1 for raw mortality data. Source data are provided as a Source Data file. c Lack of lifespan effect by daf-12 in daf-2 p sub-population lifespan in fln-2(+) background. See Supplementary data 1 for raw mortality data

(ot611) significantly reduced daf-2(m41) p lifespan (Fig. 4c; Supplementary Table 10). We therefore conclude that while daf12 does partially suppress the daf-2(m41) Age, it acts solely by increasing $\mathrm{P}$ frequency, an effect that appeared greater due to the presence of $f l n-2($ ot611) in the daf-2 mutant. The fact that the lifespans of daf-2 and daf-2; fln-2 nematodes were similar was due to antagonistic effects of $f l n-2$ on $\mathrm{P}$ frequency (increasing lifespan) and $\mathrm{p}$ survival (reducing lifespan) in this condition. These results further illustrate how $f l n-2$ variation can confound studies of lifespan genetics.

fln-2(ot611) masks eat-2 Age effect. Dietary restriction (DR) increases healthspan and lifespan in many model organisms ${ }^{34}$. In C. elegans, some Eat mutants with reduced feeding rate (e.g. eat2) show increased lifespan, which has been interpreted as resulting from $\mathrm{DR}^{35}$. In previous work in this lab with eat-2 alleles crossed into an N2M background, we often saw little effect of eat-2 on lifespan (A. Benedetto, M. Keaney, J.M.A. Tullet, and
D. Gems, unpublished). More recently we showed that many Eat mutants show reduced $\mathrm{P}$ frequency, which may reflect reduced mechanical damage to the pharynx otherwise caused by high pumping rate in early life ${ }^{9}$. Mortality deconvolution of eat-2 populations showed that the lifespan increase is largely attributable to reduced $\mathrm{P}$ death frequency. This predicts that eat-2 should increase lifespan more in a N2H background than a N2M background, due to the higher $\mathrm{P}$ frequency in the former. To test this, we performed mortality deconvolution on eat-2(ad1116) mutants in $f \ln -2(+)$ and $f \ln -2$ (ot611) backgrounds. As expected, $\mathrm{P}$ death frequency was reduced by either $f n-2$ or eat-2 (Fig. 5a). Notably, eat-2 increased lifespan more in a $f h-2(+)$ background than in a $f l n-2$ (ot611) background (mean lifespan $+27 \%$ and $+9 \%, p<0.0001, p=0.0043$, respectively, log rank test) (Fig. 5b; Supplementary Table 11), while the effect on $\mathrm{p}$ lifespan was modest in both backgrounds $(+8 \%$ and $+5 \%, p=0.0183$ and 0.0149 , respectively, log rank test) (Fig. 5c). When $\mathrm{P}$ was eliminated using antibiotics, effects of $f l n-2$ genetic background disappeared, as expected (Fig. 5d). 

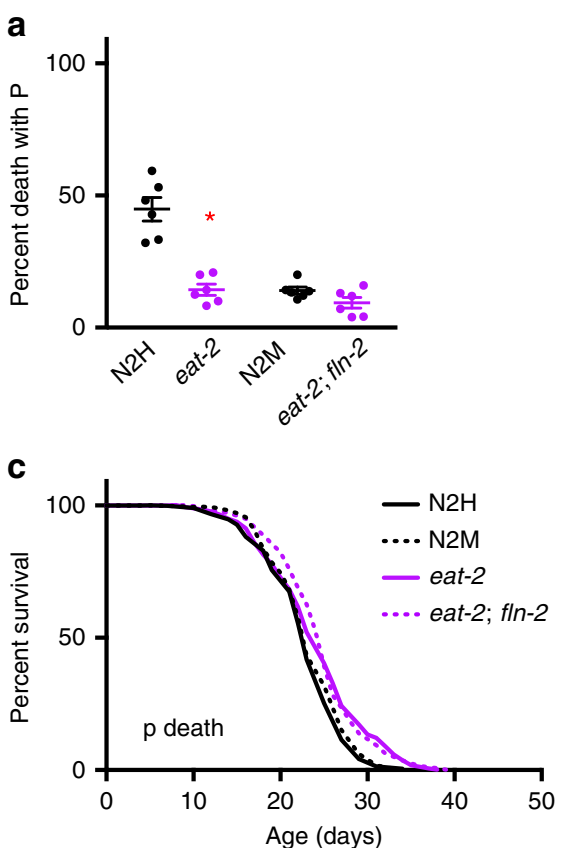

b

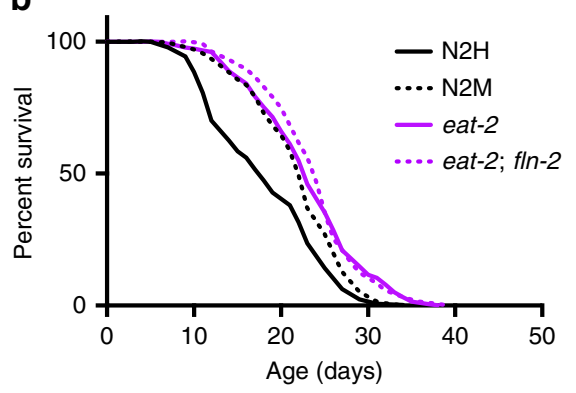

d

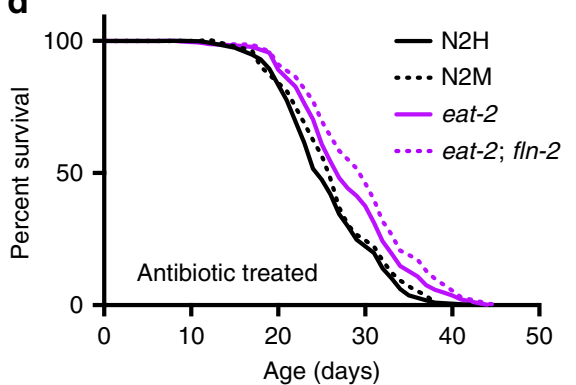

Fig. 5 Reduction of $\mathrm{P}$ death by fIn-2(ot611) masks lifespan effects of eat-2. a Necropsy analysis and $\mathbf{b}$ lifespan of eat-2(ad1116) mutants in fln-2(+) or fln-2 (ot611) backgrounds. N2H survival curve indicated by solid black line, N2M by dashed black line, eat-2 by solid purple line, and eat-2 fln-2 by dashed purple line. Error bars represent s.e.m. of three trials. See Supplementary Table 11 for full statistics, and Supplementary data 1 for raw mortality data. Source data are provided as a Source Data file. c eat-2(ad1116) causes a small but significant increase in $p$ sub-population lifespan in both fln-2(+) and fln-2(ot611) backgrounds. See Supplementary data 1 for raw mortality data. $\mathbf{d}$ Effects of fln-2(ot611) on lifespan in eat-2(ad1116) mutants disappears after treatment with antibiotics $(200 \mu \mathrm{g} / \mathrm{ml}$ carbenicillin). See Supplementary data 1 for raw mortality data

\section{Discussion}

The findings reported here reveal a major confounding variable in studies of ageing in C. elegans: variation at the $f l n-2$ locus between two widely used $\mathrm{N} 2$ lines. We demonstrate how this has actually confounded research on C. elegans ageing, distorting experimental results, and causing confusion and controversy, issues that can now be readily resolved. These results also provide further evidence of the power of mortality deconvolution and, more broadly, pathology-centred approaches to solve complex problems in biogerontology.

The advance of science often requires a process of over-coming limitations within a field, both conceptual and technical. The latter includes identification and control of confounding variables, which, over time, can lead to accumulation of unsolved questions and conflicting results. This is a particular problem with model organism biogerontology, since lifespan as a trait is particularly sensitive to uncontrolled variables ${ }^{36,37}$. For C. elegans, these include variables affecting genetic background, such as that between $\mathrm{N} 2 \mathrm{H}$ and $\mathrm{N} 2 \mathrm{M}$, and effects of variation at $f l n-2$ as described here. It also includes a range of environmental variables; since the introduction of a standardised C. elegans plate culture system ${ }^{10}$, several modifications have been introduced, including the use of FUDR to block progeny production, and antibiotics or UV irradiation to block $E$. coli proliferation. It is becoming increasingly clear that the effects of many interventions that alter $C$. elegans lifespan reflect, at least partially, changed interactions between C. elegans and E. coli $i^{9,38,39}$. Thus, variables affecting $C$. elegans-E. coli interactions, including $f l n-2$ allele variation and use of different culture conditions, either introduce confounding variables, or make it difficult to relate results from different research groups to one another.

Variation at $f l n-2$ can confound studies of the genetics of lifespan, as illustrated here using three cases, involving sir-2.1, daf-2 and daf-12, and eat-2. It is therefore possible that variation at $f l n-2$ has also confounded other studies of C. elegans ageing.
Our results resolve a controversy surrounding effects of sir-2.1 over-expression in C. elegans lifespan ${ }^{21,22,40}$. This provides a useful lesson in how science can be impeded by confounding variables, in this case three of them. The first was the presence of a $d y f$ mutation which increased lifespan in a sir-2.1(oe) $\operatorname{strain}^{21,22}$. The second was the presence of $f l n-2$ (ot611) in strains backcrossed with $\mathrm{N} 2 \mathrm{M}$, which suppressed the effect of sir-2.1(oe) on lifespan ${ }^{21}$. The third was the use of FUDR, which as we show here is required for sir-2.1(oe) to increase lifespan; it is notable that FUDR-dependent induction of increased lifespan by hypertonic stress is partially sirtuin dependent ${ }^{24}$. Does this FUDRdependence invalidate the conclusion that sir-2.1(oe) protects against ageing? This question raises important issues about the use of C. elegans as a model for understanding human ageing. We have argued elsewhere ${ }^{41,42}$ for a reconceptualization of the relationship between ageing and lifespan, as follows. Lifespan is not a read out of one process, i.e. ageing, but rather a function of some (but not all) senescent pathologies that arise as animals age. Importantly, the identity of the pathologies that limit lifespan will vary not only between species, but within species depending upon genotype and environment ${ }^{41,42}$. According to this view, the key objective for understanding ageing is to understand the etiologies of any pathology, whether they affect lifespan or not. In terms of sir-2.1(oe), FUDR and lifespan, if the use of FUDR enables studies that yield useful insights about senescent pathology, then this is a good model to study.

We showed here that under standard culture conditions (proliferating E. coli, no FUDR), previously reported suppression of daf-2( $m 41)$ Age $\left(25^{\circ} \mathrm{C}\right)$ by daf-12( $\left.m 20\right)$ is likely to have been partially attributable to variation at $f l n-2$ (Fig. $4 \mathrm{a}-\mathrm{c}$ ). There is other evidence that signalling via the DAF-9/DAF-12 pathway can promote daf-2 Age. For example, at $22.5^{\circ} \mathrm{C}$ daf-9(rh50) shortens daf-2(e1368) lifespan ${ }^{43}$, and daf-2(e1368) Age can be enhanced by treatment with exogenous DAF-12 ligand $\left(\Delta^{4} \text {-dafachronic acid }\right)^{44}$. However, our results imply that clearer 
understanding of how the IIS and DAF-12 steroid signalling pathways interact to affect ageing could be achieved by enhanced control of confounding variables, including $f l n-2$ and culture condition variables (as listed above).

These results reveal that the greater lifespan of $\mathrm{N} 2 \mathrm{M}$ is attributable to $f l n-2(o t 611)$. The presence of the $f l n-2(+)$ allele in $\mathrm{N} 2 \mathrm{H}$ and all C. elegans wild isolates examined demonstrates that $\mathrm{N} 2 \mathrm{H}$ is the wild type and $\mathrm{N} 2 \mathrm{M}$ a long-lived mutant, in contrast to a previous suggestion ${ }^{8}$. This is an argument for discontinuing the use of N2M as wild type for most purposes. The mechanisms by which $f l n$-2(ot611) protects against pharyngeal infection remain to be explored. Comparison between the two N2 strains raised on fluorescent E. coli suggests that $f l n-2($ ot611) is resistant to cuticle perforation and/or bacteria invasion. The presence of FLN-2 protein in the pharyngeal cuticle ${ }^{13}$ is consistent with a role as a direct, cell autonomous determinant of susceptibility to mechanical damage and/or bacterial invasion.

\begin{abstract}
Methods
Culture methods and strains. C. elegans maintenance was performed using standard protocols ${ }^{10}$. Strains were grown at $20^{\circ} \mathrm{C}$ on NGM agar plates seeded with E. coli OP50. C. elegans strains used: $\mathrm{N} 2$ and $\mathrm{N} 2$ male stocks recently obtained from the CGC (designated N2H and N2M, respectively), CF1038 daf-16(mu86), RB1240 $f l n-2(o k 1305 o t 611)$, FX4687 fln-2(tm4687), UN1066 xbEx0816 [fln-2c::gfp], GA1947 fln-2(syb202), GA468 fln-2(ot611) geIs3 [sir-2.1(+)+rol-6(su1006)], GA707 fln-2(ot611) wuEx166 [rol-6(su1006)], LG394 geIs3 [sir-2.1(+)+rol-6 (su1006)], LG398 geIs101 [rol-6(su1006)], NL3908 unc-119(ed3) pkIs1641(unc-119 $[+])$, NL3909 unc-119(ed3) pkIs1642(unc-119[+] sir-2.1[+]), GA1934 sir-2.1 (ok434), GA1928 daf-2(e1370), DR1296 daf-2(e1370) daf-12(m20), GA1945 daf-2 (m41), DR1547 daf-2(m41) daf-12(m20), DA1116 eat-2(ad1116), GA66 eat-2 (ad1116) $f l n$-2(ot611). Strains genotyped with respect to $f l n-2$ allele are listed in Supplementary Table 3.
\end{abstract}

Lifespan assays and necropsy analysis. Unless otherwise specified, lifespan assays were conducted at $20^{\circ} \mathrm{C}$ and without FUDR, and animals were transferred daily during the egg laying period, and every 3-7 days thereafter. Animals lost due to causes other than senescence (e.g. desiccation on the side of the plate, internal hatching, extensive rupture through the vulva) were censored. For lifespans performed at $25^{\circ} \mathrm{C}$ (daf-2 daf- 12 experiments), worms were raised at $20^{\circ} \mathrm{C}$, and transferred to $25^{\circ} \mathrm{C}$ at $\mathrm{L} 4$ stage. For trials performed in the presence of FUDR, L4 stage animals were transferred to seeded NGM plates containing the specified concentration of FUDR, and animals were transferred every 3-7 days to avoid hypertonic stress ${ }^{24}$. For antibiotic treatment, $200 \mu \mathrm{g} / \mathrm{ml}$ carbenicillin was added to two-day old lawns of OP50, and nematodes were transferred to antibiotic plates as L4 larvae. Mortality and pharyngeal status of corpses were scored every 2-3 days. For lifespan analysis on UV-irradiated E. coli OP50, bacteria were inoculated onto NGM plates and allowed to grow overnight at room temperature before exposure to UV light (Stratagene UV Stratalinker 2400) for $5 \mathrm{~min}$, 2 days prior to use (to detect and exclude plates with bacterial growth). Bacteria-free (alkaline hypochlorite-treated) C. elegans eggs were then plated on irradiated E. coli lawns. Lifespan assays were repeated at least twice, and raw mortality data are provided in the Ziehm tables (Supplementary Data 1). Survival plots were generated in GraphPad Prism using combined lifespan data from multiple trials, with L4 as day 0 on the time scale.

OP50-RFP infection analysis. An overnight culture of OP50-RFP in LB supplemented with tetracycline was washed three times in M9 to remove tetracycline, and seeded onto NGM plates two days before use. L4 worms cultured on OP50 bacteria were transferred to OP50-RFP plates, and transferred daily during the reproductive period. Before imaging, live worms were mounted on $2 \%$ agar pads and anesthetised using $0.2 \%$ levamisole. Fluorescence within pharyngeal tissue were scored at 630x magnification using a Zeiss Axiolmager Z2 microscope with a rhodamine filter. A different cohort of worms was imaged for each time point so as to avoid recovering worms from levamisole.

Test cross to confirm single-locus association. Strain GR1307 daf-16(mgDf50) was backcrossed with either N2H or N2M males, and F1s allowed to self. Single F2 animals were picked at random and the daf- 16 allele genotyped, and F3 populations of single daf-16 homozygous animals were scored in necropsy analysis and used for further backcrossing. The same analyses were performed for a single F4, F6, and F8 animal of each F2 line. All progeny of the backcrossed F2, F4, F6, and F8 animals used in lifespan were frozen and subsequently genotyped with respect to the $f \ln -2$ allele.
Whole genome sequencing. Genomic DNA was extracted using Gentra Puregene Tissue Kit (Qiagen), and sequenced with an average coverage of $100 \times$ (Center for Applied Genomics, The Hospital for Sick Children, Toronto, Canada). N2M genome sequence is available on ENA with the accession number PRJEB34655 [https://www.ebi.ac.uk/ena/browser/view/PRJEB34655], and will be incorporated in the $\mathrm{N} 2$ genome sequence on CeNDR ${ }^{14}$. For variant discovery mapping, N2H hermaphrodites and N2M males were allowed to mate overnight, after which males were removed. F1 hermaphrodites were allowed to self-fertilise, and a total of 250 F2 animals were singled and allowed to self-fertilise. From each plate, 50-60 F3 progeny were transferred to plates containing $10 \mu \mathrm{M}$ FUDR, allowed to age, and then scored for pharyngeal phenotype. 50 of the populations displaying lowest frequency of $\mathrm{P}$ death were re-analysed and 25 populations that showed less than $20 \%$ P death in both tests were selected. F3 and F4 genomic DNA from selected populations were then extracted and pooled to be sequenced. The sequencing data were processed in CloudMap using the variant discover mapping method, which mapped the causal variant to the vicinity of the $10 \mathrm{Mb}$ position on chromosome $\mathrm{X}$.

Genotyping fln-2 alleles. The following primers were used to amplify the genomic region flanking the $f l n-2$ mutation site: forward 5'-GGTGTTCGATTCTGGTCT GG-3'; reverse 5'-ACATCGACGAGAAGACAACAC-3'. PCR product was sequenced using primer $5^{\prime}$-TGTACCCAGAAATTGACAAGATAC- $3^{\prime}$. Allelespecific PCR can be used to discriminate between the genotypes, using the following primers: wild-type-specific forward 5 -taccattccgagcttattgattgttacctGGACG GCGCTGGTCCATAC-3'; ot611-specific forward 5'-GGACGGCGCTGGTCTAT AA-3'; ASP reverse 5' - ATCGCATGAACCATAAATGATG-3'; the wild-type PCR product is 30 nt longer than the mutant product.

fln-2(syb202) Y800* allele. The allele harbouring the $\mathrm{Y}^{800^{*}}$ mutation was generated using co-CRISPR method in N2H (Sunybiotech). sgRNAs TCATTCACTCCGGACGGCGCTGG and CCACGTTCTTTTTAACAGAATGG were used, and synonymous mutations were made in the sgRNA/PAM site. The final mutated sequence is tcattcactccggacggAgctggtcaatAAaaaatAcaTgttcttttaacagaatgg.

Statistics. Survival analyses of whole populations (i.e. P and p deaths) were conducted using GraphPad Prism, including right censoring of animals lost due to causes other than senescence. To test for statistically significant differences between survival curves, the non-parametric log rank test was used. In the case of $\mathrm{P}$ or $\mathrm{p}$ subpopulations, due to the absence of information about corpse type, deaths due to causes other than senescence were not censored but rather excluded altogether from statistical analysis. Sample sizes and statistics for each experiment are provided in the lifespan tables in the Supplementary file. An unpaired Student's $t$ test was used to compare frequencies of $\mathrm{P}$ death between different strains and/or conditions.

Reporting summary. Further information on research design is available in the Nature Research Reporting Summary linked to this article.

\section{Data availability}

All statistics relating to survival analysis are provided in the Supplementary tables. Raw mortality data are provided in Ziehm tables ${ }^{9}$ in Supplementary Data 1. Source data relating to mortality analysis are provided as a Source Data file. N2M genome sequence is available on ENA with the accession number PRJEB34655.

Received: 14 December 2018; Accepted: 15 October 2019; Published online: 08 November 2019

\section{References}

1. Kenyon, C. J. The genetics of ageing. Nature 464, 504-512 (2010).

2. Bitto, A., Wang, A., Bennett, C. \& Kaeberlein, M. Biochemical genetic pathways that modulate aging in multiple species. Cold Spring Harb. Perspect. Med. 5, a025114 (2015).

3. Kenyon, C., Chang, J., Gensch, E., Rudner, A. \& Tabtiang, R. A. C. elegans mutant that lives twice as long as wild type. Nature 366, 461-464 (1993).

4. Vellai, T. et al. Genetics: influence of TOR kinase on lifespan in C. elegans. Nature 426, 620 (2003).

5. Weber, K. P. et al. Whole genome sequencing highlights genetic changes associated with laboratory domestication of C. elegans. PloS one 5, e13922 (2010).

6. Sterken, M. G., Snoek, L. B., Kammenga, J. E. \& Andersen, E. C. The laboratory domestication of Caenorhabditis elegans. Trends Genet.: TIG 31, 224-231 (2015)

7. Large, E. E. et al. Selection on a subunit of the NURF chromatin remodeler modifies life history traits in a domesticated strain of Caenorhabditis elegans. PLoS Genet. 12, e1006219 (2016). 
8. Gems, D. \& Riddle, D. L. Defining wild-type life span in Caenorhabditis elegans. J. Gerontol. Ser. A, Biol. Sci. Med. Sci. 55, B215-B219 (2000).

9. Zhao, Y. et al. Two forms of death in ageing Caenorhabditis elegans. Nat. Commun. 8, 15458 (2017)

10. Brenner, S. The genetics of Caenorhabditis elegans. Genetics 77, 71-94 (1974).

11. Kumar, S. et al. Lifespan extension in C. elegans caused by bacterial colonization of the intestine and subsequent activation of an innate immune response. Dev. Cell 49, 100-117.e106 (2019).

12. Minevich, G., Park, D. S., Blankenberg, D., Poole, R. J. \& Hobert, O. CloudMap: a cloud-based pipeline for analysis of mutant genome sequences. Genetics 192, 1249-1269 (2012).

13. DeMaso, C. R., Kovacevic, I., Uzun, A. \& Cram, E. J. Structural and functional evaluation of C. elegans filamins FLN-1 and FLN-2. PloS one 6, e22428 (2011).

14. Cook, D., Zdraljevic, S., Roberts, J. \& Andersen, E. CeNDR, the Caenorhabditis elegans natural diversity resource. Nucleic Acids Res. 45, D650-D657 (2017)

15. Sarin, S. et al. Analysis of multiple ethyl methanesulfonate-mutagenized Caenorhabditis elegans strains by whole-genome sequencing. Genetics $\mathbf{1 8 5}$, 417-430 (2010).

16. C. elegans Deletion Mutant Consortium. Large-scale screening for targeted knockouts in the Caenorhabditis elegans genome. G3 (Bethesda, Md.) 2 1415-1425 (2012).

17. Hunt-Newbury, R. et al. High-throughput in vivo analysis of gene expression in Caenorhabditis elegans. PLoS Biol. 5, e237 (2007).

18. Kaeberlein, M., McVey, M. \& Guarente, L. The SIR2/3/4 complex and SIR2 alone promote longevity in Saccharomyces cerevisiae by two different mechanisms. Genes Dev. 13, 2570-2580 (1999).

19. Tissenbaum, H. A. \& Guarente, L. Increased dosage of a sir-2 gene extends lifespan in Caenorhabditis elegans. Nature 410, 227-230 (2001).

20. Viswanathan, M., Kim, S. K., Berdichevsky, A. \& Guarente, L. A role for SIR2.1 regulation of ER stress response genes in determining C. elegans life span. Developmental cell 9, 605-615 (2005).

21. Burnett, C. et al. Absence of effects of Sir2 overexpression on lifespan in C. elegans and Drosophila. Nature 477, 482-485 (2011).

22. Viswanathan, M. \& Guarente, L. Regulation of Caenorhabditis elegans lifespan by sir-2.1 transgenes. Nature 477, E1-E2 (2011).

23. Aitlhadj, L. \& Sturzenbaum, S. R. The use of FUdR can cause prolonged longevity in mutant nematodes. Mechanisms Ageing Dev. 131, 364-365 (2010).

24. Anderson, E. N. et al. C. elegans lifespan extension by osmotic stress requires FUdR, base excision repair, FOXO, and sirtuins. Mechanisms ageing Dev. 154, 30-42 (2016).

25. Burnaevskiy, N. et al. Reactivation of RNA metabolism underlies somatic restoration after adult reproductive diapause in C. elegans. eLife 7, https://doi. org/10.7554/eLife.36194 (2018).

26. Van Raamsdonk, J. M. \& Hekimi, S. FUdR causes a twofold increase in the lifespan of the mitochondrial mutant gas-1. Mechanisms ageing Dev. 132, 519-521 (2011).

27. Scott, T. et al. Host-microbe co-metabolism dictates cancer drug efficacy in C. elegans. Cell 169, 442-456 (2017).

28. Wang, Y. \& Tissenbaum, H. A. Overlapping and distinct functions for a Caenorhabditis elegans SIR2 and DAF-16/FOXO. Mechanisms ageing Dev. 127, 48-56 (2006).

29. Greiss, S., Hall, J., Ahmed, S. \& Gartner, A. C. elegans SIR-2.1 translocation is linked to a proapoptotic pathway parallel to cep-1/p53 during DNA damageinduced apoptosis. Genes Dev. 22, 2831-2842 (2008).

30. Miyata, S., Begun, J., Troemel, E. \& Ausubel, F. DAF-16-dependent suppression of immunity during reproduction in Caenorhabditis elegans. Genetics 178, 903-918 (2008).

31. Larsen, P. L., Albert, P. S. \& Riddle, D. L. Genes that regulate both development and longevity in Caenorhabditis elegans. Genetics $\mathbf{1 3 9}$ 1567-1583 (1995).

32. Gems, D. et al. Two pleiotropic classes of daf-2 mutation affect larval arrest, adult behavior, reproduction and longevity in Caenorhabditis elegans. Genetics 150, 129-155 (1998).

33. Dumas, K., Guo, C., Shih, H.-J. \& Hu, P. Influence of steroid hormone signaling on life span control by Caenorhabditis elegans insulin-like signaling. G3 3, 841-850 (2013).

34. Kapahi, P., Kaeberlein, M. \& Hansen, M. Dietary restriction and lifespan: Lessons from invertebrate models. Ageing Res. Rev., https://doi.org/10.1016/j. arr.2016.12.005 (2016).

35. Lakowski, B. \& Hekimi, S. The genetics of caloric restriction in Caenorhabditis elegans. Proc. Natl. Acad. Sci. USA 95, 13091-13096 (1998).
36. Partridge, L. \& Gems, D. Benchmarks for ageing studies. Nature 450, 165-167 (2007).

37. Lucanic, M. et al. Impact of genetic background and experimental reproducibility on identifying chemical compounds with robust longevity effects. Nat. Commun. 8, 14256 (2017).

38. Podshivalova, K., Kerr, R. \& Kenyon, C. How a mutation that slows aging can also disproportionately extend end-of-life decrepitude. Cell Rep. 19, 441-450 (2017).

39. Cabreiro, F. et al. Metformin retards aging in C. elegans by altering microbial folate and methionine metabolism. Cell 153, 228-239 (2013).

40. Couzin-Frankel, J. Genetics. Aging genes: the sirtuin story unravels. Sci. (New Y., N. Y.) 334, 1194-1198 (2011).

41. Ezcurra, M. et al. C. elegans eats its own intestine to make yolk leading to multiple senescent pathologies. Curr. Biol.: CB 28, 2544-2556 (2018).

42. Gems, D. The aging-disease false dichotomy: understanding senescence as pathology. Front. Genet. 6, 212 (2015).

43. Gerisch, B., Weitzel, C., Kober-Eisermann, C., Rottiers, V. \& Antebi, A. A hormonal signaling pathway influencing C. elegans metabolism, reproductive development, and life span. Developmental cell 1, 841-851 (2001)

44. Gerisch, B. et al. A bile acid-like steroid modulates Caenorhabditis elegans lifespan through nuclear receptor signaling. Proc. Natl Acad. Sci. USA 104, 5014-5019 (2007).

\section{Acknowledgements}

The authors thank the Caenorhabditis Genetics Center, which is funded by NIH Office of Research Infrastructure Programs (P40 OD010440), for strains used in this study, and A. Daul at the CGC for information. We thank the Mitani Lab National BioResource Project for strains. The authors thank E. Cram, H. Richly and B. Tursun for strains and reagents This work was supported by a Wellcome Trust Strategic Award to D. Gems (098565/Z/ 12/Z) and a Wellcome Trust Research Career Development Fellowship to R.J. Poole (095722/Z/11/Z). The authors would also like to thank A. Benedetto for critically reading the manuscript.

\section{Author contributions}

D.G. and Y.Z. conceived the study. H.W. and Y.Z. performed the experiments, analysed and interpreted the data. R.P. performed the Variant Discovery Mapping. D.G. and Y.Z. wrote the manuscript.

\section{Competing interests}

The authors declare no competing interests.

\section{Additional information}

Supplementary information is available for this paper at https://doi.org/10.1038/s41467 019-13062-z.

Correspondence and requests for materials should be addressed to D.G.

Peer review information Nature Communications thanks Alexander Mendenhall and other anonymous reviewer(s) for their contribution to the peer review of this work. Peer reviewer reports are available.

Reprints and permission information is available at http://www.nature.com/reprints

Publisher's note Springer Nature remains neutral with regard to jurisdictional claims in published maps and institutional affiliations.

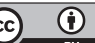

Open Access This article is licensed under a Creative Commons Attribution 4.0 International License, which permits use, sharing adaptation, distribution and reproduction in any medium or format, as long as you give appropriate credit to the original author(s) and the source, provide a link to the Creative Commons license, and indicate if changes were made. The images or other third party material in this article are included in the article's Creative Commons license, unles indicated otherwise in a credit line to the material. If material is not included in the article's Creative Commons license and your intended use is not permitted by statutory regulation or exceeds the permitted use, you will need to obtain permission directly from the copyright holder. To view a copy of this license, visit http://creativecommons.org/ licenses/by/4.0/.

(c) The Author(s) 2019 